\title{
THE GAUSSIAN-WAHL MAP FOR TRIGONAL CURVES
}

\author{
JAMES N. BRAWNER
}

(Communicated by Eric Friedlander)

\begin{abstract}
If a curve $C$ is embedded in projective space by a very ample line bundle $L$, the Gaussian map $\Phi_{C, L}$ is defined as the pull-back of hyperplane sections of the classical Gauss map composed with the Plücker embedding. When $L=K$, the canonical divisor of the curve $C$, the map is known as the Gaussian-Wahl map for $C$. We determine the corank of the GaussianWahl map to be $g+5$ for all trigonal curves (i.e., curves which admit a 3-to-1 mapping onto the projective line) by examining the way in which a trigonal curve is naturally embedded in a rational normal scroll.
\end{abstract}

\section{INTRODUCTION}

(1.1) If $L$ is a line bundle on a projective variety $X$, we can define the Gaussian map

$$
\Phi_{L}: \Lambda^{2} H^{0}(X, L) \rightarrow H^{0}\left(X, \Omega_{X}^{1} \otimes L^{2}\right)
$$

essentially by $\Phi_{L}(f \wedge g)=f d g-g d f$. The name Gaussian derives from the case where $X$ is a curve and $L$ is very ample; in this case $\Phi_{L}$ is simply the pull-back of hyperplane sections of the classical Gauss map, after composing with the Plücker embedding. When $X$ is a curve and $L=K_{X}$, the canonical divisor of the curve, Wahl proved the following remarkable theorem concerning the map $\Phi_{K}$, which we refer to as the Gaussian-Wahl map.

Theorem 1.2 (Wahl [Wa]). If a non-singular curve lies on a $K 3$ surface, then its Gaussian-Wahl map $\Phi_{K}$ is not surjective.

Other authors have shown that the Gaussian-Wahl map is surjective for the general curve of sufficiently high genus [CHM], and have studied the corank of the map for certain families of curves for which the map is not surjective. Ciliberto and Miranda have shown that $\operatorname{corank}\left(\Phi_{K}\right)=g+5$ for the general trigonal curve of genus $g \geq 4$ [CM]. They went on to exhibit a specific class of cyclic trigonal curves, defined by the equation $y^{3}=x^{3 L}-1$, for which the corank of $\Phi_{K}$ is equal to this same generic value. We prove in this paper that in fact this is the case for every trigonal curve of genus $g \geq 4$. After some preliminary discussion we prove the main theorem in $\S 3$.

Received by the editors September 9, 1993; the contents of this paper were presented at the Joint Mathematics Meetings of the AMS on January 14, 1994.

1991 Mathematics Subject Classification. Primary 14H45; Secondary 14J26. 
Theorem 1.3. The corank of the Gaussian-Wahl map is $g+5$ for every nonsingular trigonal curve of genus $g \geq 4$.

\section{TRIGONAL CURVES}

(2.1) In this section we recall several well-known facts about trigonal curves and rational normal scrolls which we will use in the proof of the main theorem (cf. [Co, Ma]). A non-singular trigonal curve $C$ of genus $g$, canonically embedded in $\mathbf{P}^{g-1}$, lies on a rational normal scroll whose invariants are determined by the $g_{3}^{1}$. Specifically, if $k$ is the unique positive integer such that $h^{0}\left((k+1) g_{3}^{1}\right)=k+2$ and $h^{0}\left((k+2) g_{3}^{1}\right) \geq k+4$, then $C$ lies on the scroll $S_{k, l} \subset \mathbf{P}^{k+l+1}$, where $k+l=g-2$ and $k \leq l$. Maroni showed that this invariant $k$ is bounded by

$$
\frac{g-1}{d} \leq k+1 \leq \frac{g}{2}
$$

and that the ruling of the scroll cuts out the $g_{3}^{1}$ on the curve [Ma]. Where convenient we will denote the scroll simply as $S$.

(2.2) The scroll $S_{k, l}$, which is isomorphic to the surface $\mathbf{F}_{n}, n=l-k$, has Picard group generated by a hyperplane section $H$ and a fibre of the ruling $R$, with the following intersections: $H^{2}=k+l, H \cdot R=1$, and $R^{2}=0$. If $k<l$, there is a unique curve $B$ of negative self-intersection on the scroll with class $B \equiv H-l R$ and intersections $B^{2}=-(l-k)$ and $B \cdot R=1$. The class of the curve $C$ and the canonical divisor of the scroll $K_{S}$ are easily computed, as are those of two other divisors that we will use later:

$$
\begin{gathered}
C \equiv 3 H+(4-g) R, \\
K_{S} \equiv-2 H+(g-4) R \equiv-2 B-(n+2) R, \\
K_{S}+C \equiv H \equiv B+l R, \\
2 K_{S}+C \equiv-H+(g-4) R \equiv-B+(k-2) R .
\end{gathered}
$$

(2.3) We collect the following computations about global sections of sheaves over the scroll $S$ (cf. [DM 3.2, 3.3]). If $D \equiv r B+s R$ is a divisor on a rational normal scroll $S_{k, l} \cong \mathbf{F}_{l-k}=\mathbf{F}_{n}$, then

(i) $H^{0}\left(S, \mathscr{O}_{S}(D)\right)=0=H^{0}\left(S, \Omega_{S}^{1}(D)\right)$ if $r<0$ or $s<0$.

(ii) $h^{0}\left(S, \Omega_{S}^{1}(D)\right)=2 r s-n r^{2}-2$ if $r \geq 1$ and $s \geq n r+1$.

\section{THE PROOF OF THE THEOREM}

(3.1) If the trigonal curve $C$ has genus $g=4$, then it is a complete intersection curve, which we handle with a separate argument at the end of the proof; for the remainder, we assume $g \geq 5$. From the inclusion $C \subset S$, we have the following diagram involving the Gaussian surface map $\Phi_{S, K+C}$ :

$$
\begin{array}{ccc}
\Lambda^{2} H^{0}\left(S, K_{S}+C\right) & \stackrel{\Phi_{S, K+C}}{\longrightarrow} H^{0}\left(S, \Omega_{S}^{1}\left(2 K_{S}+2 C\right)\right) \\
\operatorname{Res} \downarrow & \gamma \downarrow \\
\Lambda^{2} H^{0}\left(C, \Omega_{C}^{1}\right) & \stackrel{\Phi_{K}}{\longrightarrow} & H^{0}\left(C, \Omega_{C}^{1 \otimes 3}\right)
\end{array}
$$


First notice that the restriction map $H^{0}\left(S, K_{S}+C\right) \rightarrow H^{0}\left(C, \Omega_{C}^{1}\right)$ is surjective because the scroll $S$ is a regular surface; consequently the left vertical map Res is surjective as well. We proceed to prove the theorem by showing that the top map is surjective and the right vertical map $\gamma: H^{0}\left(S, \Omega_{S}^{1}\left(2 K_{S}+2 C\right)\right) \rightarrow$ $H^{0}\left(C, \Omega_{C}^{1 \otimes 3}\right)$ is injective. The rank of the Gaussian-Wahl map $\Phi_{K}$ is then equal to the dimension of the space $H^{0}\left(S, \Omega_{S}^{1}\left(2 K_{S}+2 C\right)\right)$, which we easily compute to be $4 g-10$, and the theorem follows.

Proposition 3.2. $\Phi_{S, K+C}$ is surjective with rank $=4 g-10$.

Proof. Duflot and Miranda have calculated when the Gaussian map $\Phi_{S, D}$ is surjective for a divisor $D$ on a rational normal scroll $S_{k, l} \cong \mathbf{F}_{l-k}=\mathbf{F}_{n}$ by explicitly writing bases for $H^{0}\left(S, \mathscr{O}_{S}(D)\right)$ and $H^{0}\left(S, \Omega_{S}^{1}(2 D)\right)$. The first part of the proposition follows immediately from the following result [DM, 4.5].

Theorem 3.3 (Duflot-Miranda). The Gaussian surface map

$$
\Phi_{S, D}: H^{0}\left(S, \mathscr{O}_{S}(D)\right) \rightarrow H^{0}\left(S, \Omega_{S}^{1}(2 D)\right)
$$

is surjective for a divisor $D \equiv r B+s R$ on $S_{k, l} \cong \mathbf{F}_{n}$ if $r \geq 0$ and $s \geq n r+1$.

In our case, $D \equiv K_{S}+C \equiv B+l R$, so $r=1 \geq 0$ and $s=l$. If $g \geq 5$, then Maroni's inequality shows that $k \geq 1$; consequently $n r+1=l-k+1 \leq l=s$ and the theorem applies. Therefore the rank of $\Phi_{S, K+C}$ is simply the dimension of the target space, which we compute using (2.3):

$$
\begin{aligned}
\operatorname{rank}\left(\Phi_{S, K+C}\right) & =h^{0}\left(S, \Omega_{S}^{1}\left(2 K_{S}+2 C\right)\right) \\
& =h^{0}\left(S, \Omega_{S}^{1}(2 B+2 l R)\right) \\
& =2(2)(2 l)-(l-k) 4-2 \\
& =4(l+k)-2 \\
& =4 g-10
\end{aligned}
$$

since $r=2 \geq 1$ and $s=2 l \geq 2(l-k)+2$.

Proposition 3.4. $\gamma: H^{0}\left(S, \Omega_{S}^{1}\left(2 K_{S}+2 C\right)\right) \rightarrow H^{0}\left(C, \Omega_{C}^{1 \otimes 3}\right)$ is injective.

Proof. Consider the following two exact sequences associated with the log complex of $C \subset S$ :

$$
\begin{aligned}
& 0 \rightarrow \Omega_{S}^{1} \rightarrow \Omega_{S}^{1}(\log C) \rightarrow \mathscr{O}_{C} \rightarrow 0, \\
& 0 \rightarrow \Omega_{S}^{1}(\log C)(-C) \rightarrow \Omega_{S}^{1} \rightarrow \Omega_{C}^{1} \rightarrow 0 .
\end{aligned}
$$

We twist and take global sections to construct the following commutative diagram:

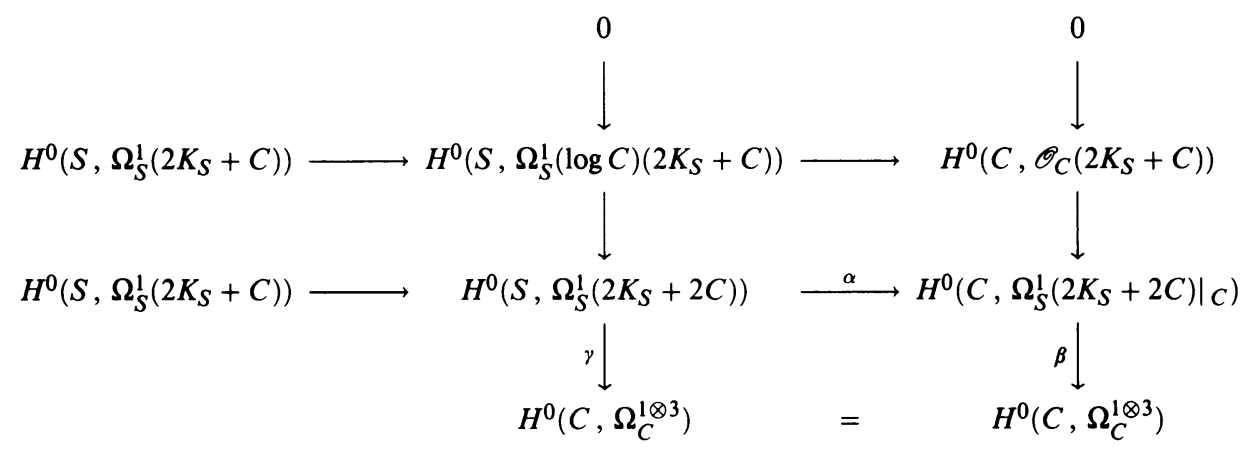


The right vertical sequence is obtained from a twist of the standard conormal sequence

$$
0 \rightarrow \mathscr{O}_{C}(-C)-\left.\Omega_{S}^{1}\right|_{C} \rightarrow \Omega_{C}^{1} \rightarrow 0 .
$$

We show that $\operatorname{ker}(\gamma)=H^{0}\left(S, \Omega_{S}^{1}(\log C)\left(2 K_{S}+C\right)\right)=0$.

(3.5) Let $\pi: S \rightarrow \mathbf{P}^{1}$ be the map associated with the $\mathbf{P}^{1}$-bundle $S, B$, and $R$ generators of $\operatorname{Pic}(S)$ as above, and let $Z$ be the 0 -dimensional subscheme of $S$ of ramification points of the restriction map $\left.\pi\right|_{C}: C \rightarrow \mathbf{P}^{1}$. Since $K_{S} \equiv-2 B-(n+2) R$ and $\Omega_{\mathbf{P}^{1}}^{1} \cong \mathscr{O}_{\mathbf{P}^{1}}(-2)$, we have the following exact sequence:

$$
0 \rightarrow \pi^{*} \Omega_{\mathbf{P}^{1}}^{1} \rightarrow \Omega_{S}^{1} \rightarrow \mathscr{O}_{S}(-2 B-n R) \rightarrow 0 .
$$

Furthermore, since $\Omega_{S}^{1}(\log C)(-C) \subset \Omega_{S}^{1}$, we can construct the following diagram involving the ideal sheaf of $Z$ :

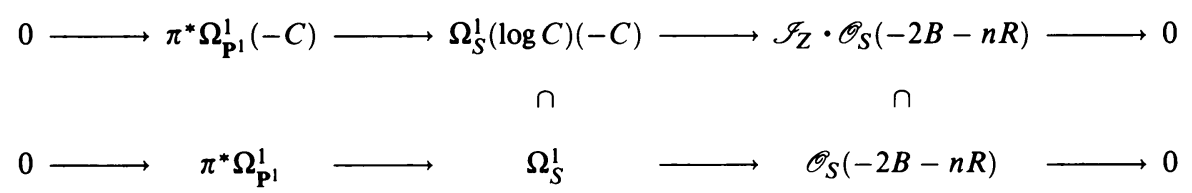

Twisting the top sequence by $2 K_{S}+2 C \equiv 2 B+2 l R$ and taking global sections gives

$$
\begin{aligned}
0 & \rightarrow H^{0}\left(S, \pi^{*} \Omega_{\mathbf{P}^{1}}^{1}\left(2 K_{S}+C\right)\right) \rightarrow H^{0}\left(S, \Omega_{S}^{1}(\log C)\left(2 K_{S}+C\right)\right) \\
& \rightarrow H^{0}\left(\mathscr{I}_{\mathbf{Z}} \cdot \mathscr{O}_{S}((g-2) R)\right) .
\end{aligned}
$$

The first space is zero since

$$
H^{0}\left(S, \pi^{*} \Omega_{\mathbf{P 1}}^{1}(-B+(k-2) R)\right) \cong H^{0}\left(S, \mathscr{O}_{S}(-B+(k-4) R)\right),
$$

which is zero by (2.3). Now, since

$$
H^{0}\left(S, \mathscr{O}_{S}((g-2) R)\right) \cong H^{0}\left(S, \pi^{*} \mathscr{O}_{\mathbf{P}^{1}}(g-2)\right)
$$

the former space can be represented by homogeneous polynomials of degree $\leq$ $g-2$ over $\mathbf{P}^{1}$, while $H^{0}\left(\mathscr{I}_{Z} \cdot \mathscr{O}_{S}((g-2) R)\right)$ is isomorphic to those polynomials which vanish on the scheme $Z$. The Riemann-Hurwitz formula shows that there are at least $g+2$ ramification points of the restriction map $\left.\pi\right|_{C}: C \rightarrow \mathbf{P}^{1}$ (with equality if all are total ramification points); but no polynomial of degree $\leq g-2$ can vanish on $\geq g+2$ points. Therefore

$$
H^{0}\left(\mathscr{I}_{Z} \cdot \mathscr{O}_{S}((g-2) R)\right)=0, H^{0}\left(S, \Omega_{S}^{1}(\log C)\left(2 K_{S}+C\right)\right)=0,
$$

and the proposition is proved.

\section{TRigonal CuRves of Genus $g=4$}

(4.1) To finish the proof of the theorem, we show that $\operatorname{corank}\left(\Phi_{K}\right)=g+5=9$ for the trigonal curves of genus 4 , each of which is the complete intersection of a quadric and a cubic surface in $\mathbf{P}^{3}$. Accordingly we construct the following 
diagram involving the Gaussian map $\Phi$ for the divisor $\mathscr{O}_{\mathbf{P}},(1)$ on $\mathbf{P}^{3}$ :

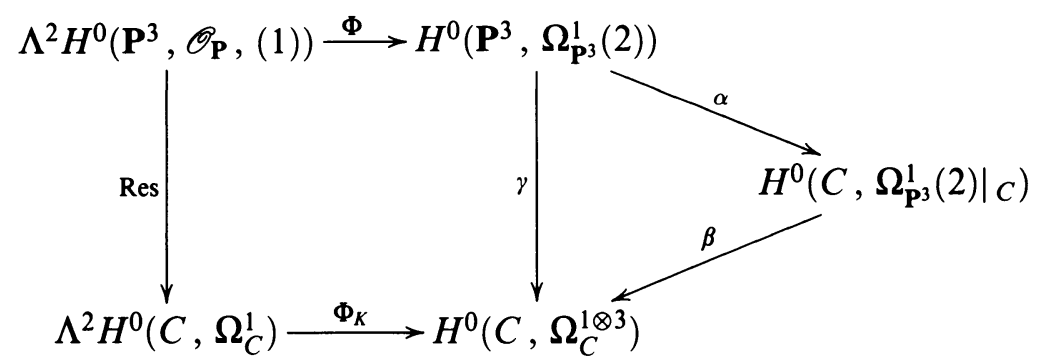

By a theorem of Wahl the Gaussian map for any divisor $\mathscr{O}_{\mathbf{P}^{n}}(k)$ on $\mathbf{P}^{n}$ is surjective $(n \geq 1)$, so the top map $\Phi$ is surjective [Wa]. The left vertical map Res is again surjective due to the normality of $\mathbf{P}^{3}$, and the right map $\gamma$ factors through the space $H^{0}\left(C, \Omega_{\mathrm{P}^{3}}^{1},\left.(2)\right|_{C}\right)$. We show that $\operatorname{Im}(\alpha) \cap \operatorname{ker}(\beta)=0$ and hence that the Gaussian-Wahl map has rank equal to the rank of the map $\alpha$ in the diagram above. We write $\left\{x_{j} d x_{i}-x_{i} d x_{j} \mid 0 \leq i<j \leq 3\right\}$ as a 6-dimensional basis for the space $H^{0}\left(\mathbf{P}^{3}, \Omega_{\mathbf{P}^{3}}^{1}(2)\right)$. An element of $\operatorname{Im}(\alpha) \cap \operatorname{ker}(\beta)$ can be expressed as a quadric polynomial $Q$ that vanishes along the curve $C$ such that

$$
d Q \equiv \sum_{0 \leq i<j \leq 3} \lambda_{i j}\left(x_{j} d x_{i}-x_{i} d x j\right) \quad\left(\bmod I_{C}\right),
$$

where $\lambda_{i j} \in \mathbf{C}$. Since $C$ is not contained in any hyperplane of $\mathbf{P}^{3}$, this means

$$
\frac{\partial Q}{\partial x_{i}}=\sum_{j>i} \lambda_{i j} x_{j}-\sum_{j<i} \lambda_{j i} x_{i} \quad \forall i,
$$

and $2 Q=0$ by Euler's lemma. Therefore, $\operatorname{Im}(\alpha) \cap \operatorname{ker}(\beta)=0, \operatorname{rank}\left(\Phi_{K}\right)=$ $\operatorname{rank}(\alpha)=6$, and $\operatorname{corank}\left(\Phi_{K}\right)=9$.

\section{REFERENCES}

[Br] J. Brawner, The Gaussian map $\Phi_{K}$ for curves with special linear series, Ph.D. dissertation, University of North Carolina, Chapel Hill, 1992.

[CHM] C. Ciliberto, J. Harris, and R. Miranda, On the surjectivity of the Wahl map, Duke Math. J. 57 (1988), 829-858.

[CM] C. Ciliberto and R. Miranda, Gaussian maps for certain families of canonical curves, Complex Projective Geometry (G. Ellingsrud et al., eds.), London Math. Soc. Lecture Note Ser., vol. 179, Cambridge Univ. Press, Cambridge and New York, 1992, pp. 106-127.

[Co] M. Coppens, The Weierstrass gap sequences of the ordinary ramification points of trigonal coverings of $\mathbf{P}^{1}$; Existence of a kind of Weierstrass gap sequence, J. Pure Appl. Algebra 43 (1986), 11-25.

[DM] J. Duflot and R. Miranda, The Gaussian map for rational ruled surfaces, Trans. Amer. Math. Soc. 330 (1992), 447-459.

[Ma] A. Maroni, Le serie lineari sulle curve trigonali, Ann. Mat. Pura Appl. 25 (1946), 341-354.

[Wa] J. Wahl, The Jacobian algebra of a graded Gorenstein singularity, Duke Math. J. 55 (1987), 843-871.

Department of Mathematics and Computer Science, St. John's University, Jamaica, NEW YORK 11439

E-mail address: brawnerjosjuvm.stjohns.edu 